\title{
Catching a New Force by the Tail
}

\author{
Simone Alioli* \\ CERN Theory Division, CH-1211, Geneva 23, Switzerland \\ and Università degli Studi di Milano Bicocca, Piazza della Scienza 3, 20126 Milan, Italy \\ Marco Farina ${ }^{\dagger}$ \\ New High Energy Theory Center, Department of Physics, Rutgers University, \\ 136 Frelinghuisen Road, Piscataway, New Jersey 08854, USA \\ Duccio Pappadopulo and Joshua T. Ruderman ${ }^{\S}$ \\ Center for Cosmology and Particle Physics, Department of Physics, New York University, New York, New York 10003, USA
}

(Received 13 December 2017; published 8 March 2018)

\begin{abstract}
The Large Hadron Collider (LHC) is sensitive to new heavy gauge bosons that produce narrow peaks in the dilepton invariant mass spectrum up to about $m_{Z^{\prime}} \sim 5 \mathrm{TeV} Z^{\prime}$ s that are too heavy to produce directly can reveal their presence through interference with standard model dilepton production. We show that the LHC can significantly extend the mass reach for such $Z^{\prime}$ s by performing precision measurements of the shape of the dilepton invariant mass spectrum. The high-luminosity LHC can exclude, with $95 \%$ confidence, new gauge bosons as heavy as $m_{Z^{\prime}} \sim 10-20 \mathrm{TeV}$ that couple with gauge coupling strength of $g_{Z^{\prime}} \sim 1-2$.
\end{abstract}

DOI: 10.1103/PhysRevLett.120.101801

Introduction.-Apart from gravity and the Higgs force, all known forces are mediated by spin-1 particles: the photon for electromagnetism, the $W / Z$ bosons for the weak force, and gluons for the strong force.

The search for new forces and their massive mediators is a well-motivated arena for both experiment and theory. New short-range Abelian gauge forces appear in many extensions of the standard model (SM) [1-22] (see also Refs. [23,24] for reviews), are an active area of investigation at the Large Hadron Collider (LHC) [25-31], and serve as standard benchmarks to test the performances of future colliders [32-39]. Additional nonanomalous $U(1)$ gauge groups [40-49] are a relatively innocuous extension of the SM, as the masses of the associated vector bosons do not require the existence of additional scalar degrees of freedom [50-52], and consequently a worsening of the hierarchy problem.

The traditional strategy to search for $Z^{\prime}$ s at colliders has been to perform "bump hunts." For $Z$ 's decaying to leptons, the dilepton invariant mass distribution is scrutinized for narrow peaks rising above the monotonically falling background. Searches at the LHC are sensitive to $Z^{\prime}$ s with masses up to about $5 \mathrm{TeV}$ [25-29].

Published by the American Physical Society under the terms of the Creative Commons Attribution 4.0 International license. Further distribution of this work must maintain attribution to the author(s) and the published article's title, journal citation, and DOI. Funded by SCOAP ${ }^{3}$.
For masses above $5 \mathrm{TeV}$, bump hunts lose sensitivity as the cross section for direct production vanishes. When the mass $M$ of the new vector boson is too large for direct production, the main contribution of the $Z^{\prime}$ at energies $E \ll M$ are interference effects [53-56], which modify the shapes of kinematical distributions. If the $Z^{\prime}$ couples to both quarks and leptons, it modifies the invariant mass distribution of Drell-Yan processes $p p \rightarrow \ell^{+} \ell^{-}, \ell=e, \mu$. The interference effects can be captured by a small number of higher-dimension operators, obtained by integrating out the $Z^{\prime}$ (see Fig. 1), and are therefore relatively insensitive to the specific details of the $Z^{\prime}$ model.

In this Letter, we assess the reach of the LHC to probe heavy $Z$ 's through precision fits to the shape of the invariant mass spectrum of dileptons. Previous studies of the interference of heavy $Z^{\prime} \mathrm{s}$ at the LHC found that a $5 \sigma$ discovery will be difficult [12] and estimated the reach of early $13 \mathrm{TeV}$ measurements [22]. We go beyond these preliminary studies by performing the first comprehensive

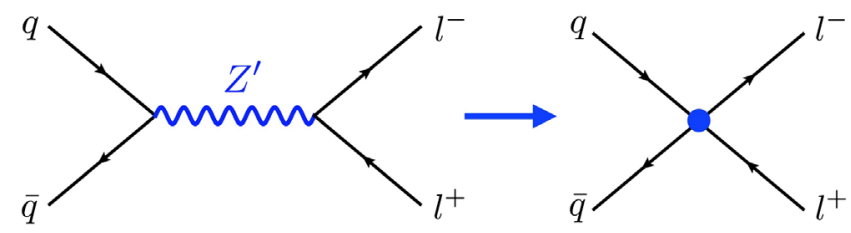

FIG. 1. At energies $E$ much smaller than the mass $M$ of the heavy gauge boson $Z^{\prime}$, the effect of the new physics on the Drell-Yan process, $p p \rightarrow \ell^{+} \ell^{-}$, is encoded by a finite set of fourfermion contact operators. 
study of theoretical uncertainties and their correlations, and by mapping the future reach of the full LHC data set. We find that a vast parameter space of $Z^{\prime} \mathrm{s}$ will be probed at the LHC. Deviations in the shape of the Drell-Yan distribution have also been used to constrain effective operators [57-60], the running of electroweak gauge couplings [61,62], and other effects from new states [63-66].

The rest of this Letter is organized as follows: We begin by reviewing the class of $Z^{\prime}$ models that we study. Then we present the reach we find of the LHC to the interference effects of heavy $Z^{\prime}$ s. We finish with our conclusions. We include Supplemental Material [67] that contains a technical description of our SM prediction, projections for future higher-energy colliders, and a comparison of our bounds with experimental contact operator bounds.

The minimal model. - A class of $Z^{\prime}$ models motivated by their simplicity and minimality has been studied in Refs. [40-49]. These minimal $Z^{\prime}$ models are defined by the requirement that the new $U(1)$ vector boson gauge a linear combination of the hypercharge $(Y)$ and the difference between baryon- and lepton-number $(B-L)$ currents. This ensures that the model is anomaly free as long as righthanded neutrinos are present. The gauge structure also ensures flavor-universal interactions for the new vector field.

The Lagrangian describing the interactions of the new vector boson $\mathcal{Z}$ can be written as

$\mathcal{L}=-\frac{1}{4} \mathcal{Z}_{\mu \nu}^{2}+\frac{M^{2}}{2} \mathcal{Z}_{\mu}^{2}-\mathcal{Z}_{\mu}\left(g_{Y} J_{H}^{\mu}+g_{Y} J_{Y}^{\mu}+g_{B L} J_{B L}^{\mu}\right)$,

where $J_{Y}^{\mu}=\sum_{f} Q_{Y}^{(f)} \bar{f} \gamma^{\mu} f$ and $J_{B L}^{\mu}=\sum_{f} Q_{B L}^{(f)} \bar{f} \gamma^{\mu} f$ are the fermionic hypercharge and $B-L$ currents, respectively, and $J_{H}^{\mu} \equiv i Q_{Y}^{(H)}\left(H^{\dagger} D^{\mu} H-D^{\mu} H^{\dagger} H\right)$. The SM field charges $Q_{Y}$ and $Q_{B L}$ are shown in Table I. The couplings $g_{Y}$ and $g_{B L}$ define the strength of the interactions between the $\mathcal{Z}$ boson and the respective currents.

The spectrum contains three neutral vector bosons: a massless photon and two massive vectors, to be identified with the $Z$ boson and the heavy $Z^{\prime}$. When $g_{Y} \neq 0$, the coupling between $\mathcal{Z}$ and the Higgs boson current leads to a mixing between the $Z$ boson and $\mathcal{Z}$. Their masses are approximately given by $m_{Z} \approx g_{Z} v / 2 \equiv m_{Z_{0}}$ and $m_{Z^{\prime}} \approx M$, with $g_{Z}^{2} \equiv g^{\prime 2}+g_{2}^{2}$ and $v=246 \mathrm{GeV}$. Corrections to these equations are small, of the order $\left(g_{Y}^{2} / g_{Z}^{2}\right)\left(m_{Z_{0}}^{2} / M^{2}\right)$, which is also the typical size of the corrections to electroweak observables. In terms of the gauge eigenstates $B, W_{3}$, and $\mathcal{Z}$,

TABLE I. Hypercharge and $B-L$ charges.

\begin{tabular}{lcccccc}
\hline \hline$f$ & $H$ & $\ell_{L}$ & $e_{R}$ & $q_{L}$ & $u_{R}$ & $d_{R}$ \\
\hline$Q_{Y}$ & $1 / 2$ & $-1 / 2$ & -1 & $1 / 6$ & $2 / 3$ & $-1 / 3$ \\
$Q_{B L}$ & 0 & -1 & -1 & $1 / 3$ & $1 / 3$ & $1 / 3$ \\
\hline \hline
\end{tabular}

$Z=\cos \alpha Z_{0}-\sin \alpha \mathcal{Z}, \quad Z^{\prime}=\sin \alpha Z_{0}+\cos \alpha \mathcal{Z}$,

where $Z_{0}$ is the unperturbed $Z$-boson wave function $Z_{0} \propto g_{2} W_{3}-g^{\prime} B$, and

$$
\tan 2 \alpha=\frac{2 g_{Y} / g_{Z} m_{Z_{0}}^{2}}{M^{2}-m_{Z_{0}}^{2}\left(1-g_{Y}^{2} / g_{Z}^{2}\right)} \approx 2 \frac{g_{Y}}{g_{Z}} \frac{m_{Z_{0}}^{2}}{M^{2}} .
$$

The couplings of the physical vector bosons to SM fermions are

$$
\begin{aligned}
J_{Z}^{\mu} & =\cos \alpha J_{Z_{0}}^{\mu}-\sin \alpha J_{\mathcal{Z}}^{\mu}, \\
J_{Z^{\prime}}^{\mu} & =\sin \alpha J_{Z_{0}}^{\mu}+\cos \alpha J_{\mathcal{Z}}^{\mu},
\end{aligned}
$$

where $J_{Z_{0}}^{\mu}$ is the $Z$-boson current in the SM, $J_{Z_{0}}^{\mu}=$ $g_{Z} \sum_{f} \bar{f} \gamma^{\mu}\left(T_{3 L}-\sin ^{2} \theta_{W} Q\right) f$, and $J_{\mathcal{Z}}^{\mu}=g_{Y} J_{Y}^{\mu}+g_{B L} J_{B L}^{\mu}$.

At energies $E \ll M$, the physics described by Eq. (1) is captured by an effective field theory (EFT) obtained by integrating out $\mathcal{Z}$. At leading order in $1 / M$, this is given by

$$
\mathcal{L}_{\mathrm{EFT}}=-\frac{1}{2 M^{2}}\left(g_{Y} J_{H}^{\mu}+g_{Y} J_{Y}^{\mu}+g_{B L} J_{B L}^{\mu}\right)^{2} .
$$

In the left panel of Fig. 2, we show the ratio of the dilepton invariant mass distribution in the presence of a $Z^{\prime}$ to the SM, and we compare the results obtained from the full theory in Eq. (1) to the EFT of Eq. (5). The two calculations agree for invariant masses within the reach of the LHC, when the $Z^{\prime}$ is heavy and not too wide.

Existing bounds and projections.-In our analysis we consider two kinds of constraints on minimal $Z^{\prime}$ models. The first set comes from low-energy measurements, including constraints from LEPI and LEPII [70,71]. These can be evaluated using the low-energy Lagrangian in Eq. (5) and depend on the parameter combinations $g_{Y} / M$ and $g_{B L} / M$. We extract these bounds from the global fit in Ref. [60].

The second set of constraints comes from the LHC measurements of the dilepton invariant mass distribution in $p p \rightarrow \ell^{+} \ell^{-}$collisions. If the $Z^{\prime}$ is light enough to be produced on shell, it will manifest as a resonant excess in the dilepton spectrum. Bump hunt searches are optimized to look for this kind of isolated excess [25-29]. The results presented in Ref. [29] are currently the strongest constraints on minimal $Z^{\prime}$ s for $M<5 \mathrm{TeV}$.

In this section, we establish the reach of the LHC for $Z^{\prime} \mathrm{s}$ that are too heavy to be efficiently produced on shell, and thus escape bump hunt searches. A $Z^{\prime}$ will distort the high energy tail of the dilepton invariant mass distribution, as shown in the left panel of Fig. 2. To project the sensitivity to $Z^{\prime}$ s, we must predict both the SM and the new physics contributions to the dilepton spectrum.

For the SM prediction, we use next-to-next-to-leadingorder (NNLO) QCD [72-77] and next-to-leading-order (NLO) electroweak (EW) [78-83] results from FEWZ-3.1 [84]. Details about the generation and the evaluation of the 

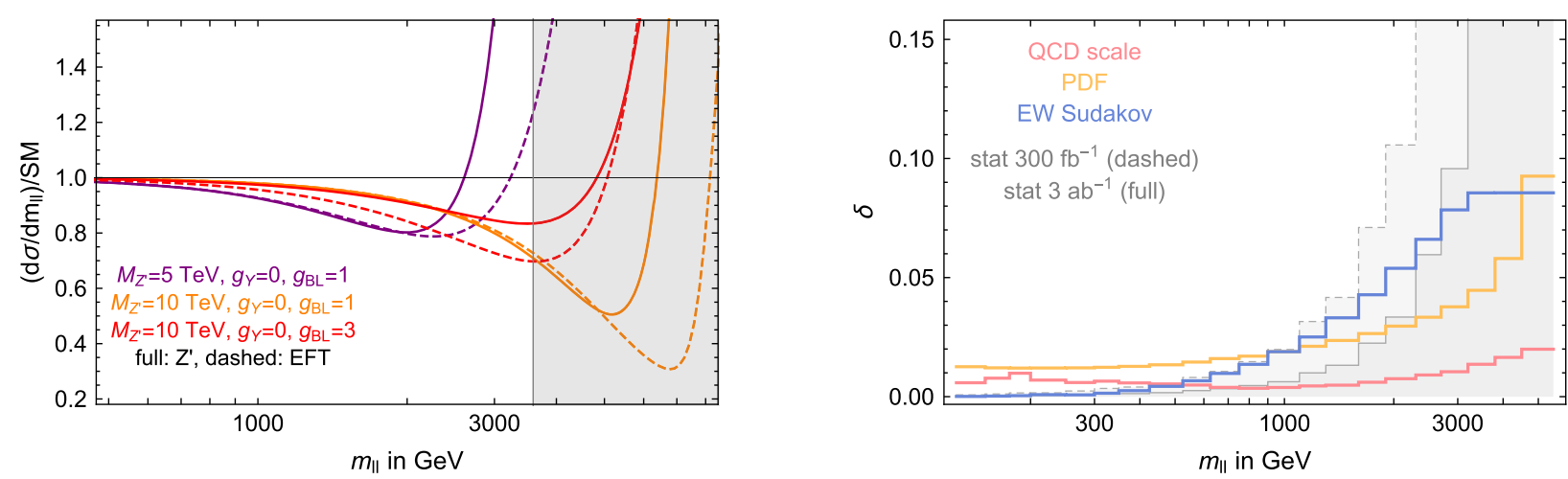

FIG. 2. Left panel: Ratio of the dilepton invariant mass distribution in the $Z^{\prime}$ model to the SM. The solid lines are calculated using the full model of Eq. (1), while the dashed lines are calculated using the effective field theory (EFT) of Eq. (5). In the gray region, there are three expected SM events with a luminosity of $3 \mathrm{ab}^{-1}$. Right panel: Systematic theoretical uncertainties used in our analysis. We also show the size of the statistical uncertainty associated with the SM prediction.

QCD and parton distribution function (PDF) uncertainties are reported in the Supplemental Material. The NLO EW corrections in FEWZ include virtual $\gamma, Z$, and $W$ exchange and real QED corrections, but do not include real $W$ or $Z$ emissions. Real $W / Z$ emissions could be important at high energies, in an inclusive measurement, and we include them after calculating them separately at leading order using MadGraph5_aMC@NLO [85] (see Ref. [86] for a previous calculation).

Our treatment of EW uncertainties, which is described in the Supplemental Material, is designed to capture the effect of missing two-loop Sudakov logarithms [86-93]. A visual summary of the various theoretical uncertainties is presented in the right panel of Fig. 2.

New physics predictions are calculated at leading order (LO) and are multiplied by the ratio of the SM NNLO QCD cross section to the SM LO one. This is justified by the fact that the relative size of the NNLO QCD corrections only depends on the invariant mass of the dilepton system. Both the SM and new physics cross sections are calculated in the dilepton invariant mass bins shown in the right panel of Fig. 2. We apply a $p_{T}>25 \mathrm{GeV}$ and $|\eta|<2.5$ cut on leptons, and we assume 65\% (80\%) identification efficiency for dielectron (dimuon) events, motivated by past LHC Drell-Yan measurements [94,95].

In order to infer the reach for minimal $Z^{\prime}$ models, we fit to the Born-level cross section, after unfolding detector effects. We perform a $\chi^{2}$ test, including QCD-scale, EW, and PDF uncertainties, and their respective correlations across different bins of invariant mass. The scale and EW uncertainties are fully correlated, whereas the PDF uncertainties exhibit nontrivial correlations. The Supplemental Material describes our $\chi^{2}$ test, and the derivation of PDF correlations, in more detail. There is also an experimental uncertainty due to unfolding, arising from effects such as detector resolution, energy scale, and lepton identification efficiency. We estimate the experimental uncertainty as being composed of uncorrelated and fully correlated components, both of which we take to be $5 \%$ of the cross section, bin by bin. This choice is motivated by the size of experimental uncertainties in previous unfolded Drell-Yan measurements conducted at $\sqrt{s}=8 \mathrm{TeV}$ [94,95].

Figure 3 shows the comparison between the low-energy bounds and projected LHC bounds on the minimal $Z^{\prime}$ models extracted using the EFT of Eq. (5). We introduce an angular variable, $\tan \theta \equiv g_{Y} / g_{B L}$, and rewrite Eq. (5) as a function of the dimensionless coefficient $R \equiv \sqrt{g_{Y}^{2}+g_{B L}^{2}} / g_{Z}$. For a given value of $\theta$, the $95 \%$ C.L. lower bound $M / R$ is shown in Fig. 3. Surprisingly, the LHC starts to be competitive with low-energy bounds around the present time, with an integrated luminosity of $50 \mathrm{fb}^{-1}$.

Notice that while the bounds extracted from the lowenergy experiments have a wide range of applicability, in terms of the mass $M$ of the resonance, the LHC bounds

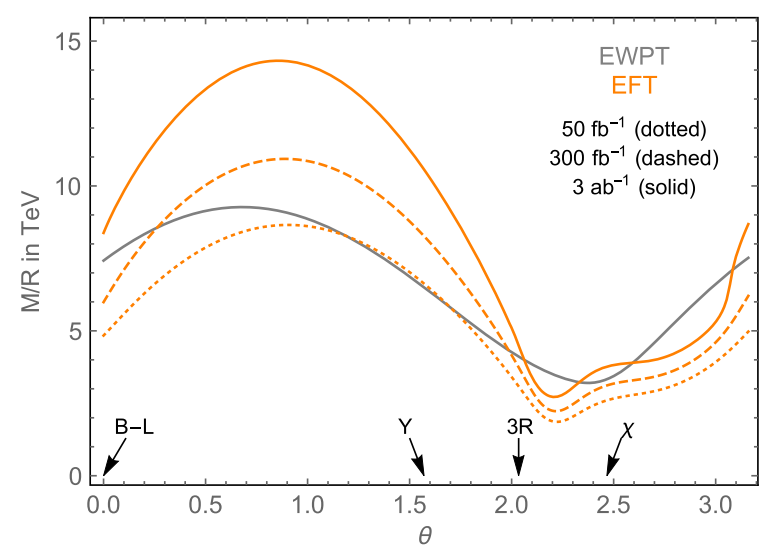

FIG. 3. $95 \%$ C.L. lower bound on $M / R$ as a function of $\theta$. The constraint coming from low-energy experiments is shown in gray, while the reach of the LHC is shown in orange. Specific models are identified: pure $B-L(\tan \theta=0)$, hypercharge $(\tan \theta=+\infty)$, $T_{3 R}(\tan \theta=-2)$, and $U(1)_{\chi}(\tan \theta=-4 / 5)[23,24]$. Notice that these relations are defined at tree level. 

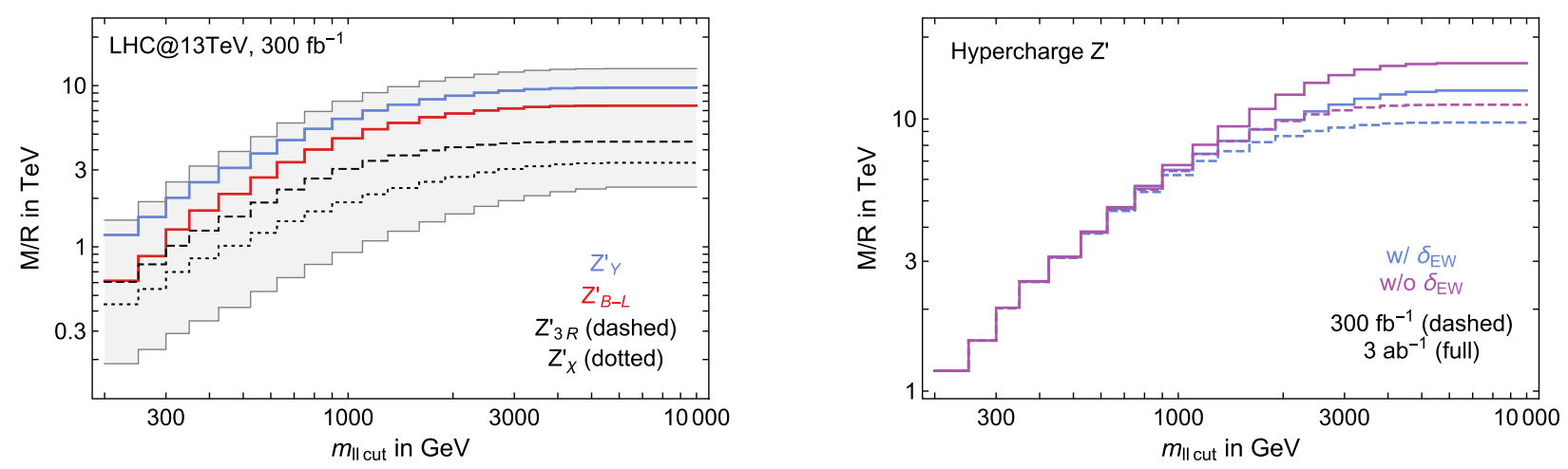

FIG. 4. Left panel: $95 \%$ C.L. lower bound on $M / R$ as a function of $m_{\ell \ell \text { cut }}$, for three example models, defined by specific choices of $\theta$ (see Fig. 3). Right panel: $95 \%$ C.L. lower bound on $M / R$ for the hypercharge model $(\theta=\pi / 2)$ as a function of $m_{\ell \ell \text { cut }}$. We show how the bound differs using two different choices for the total integrated luminosity $\left(300 \mathrm{fb}^{-1}\right.$ and $\left.3 \mathrm{ab}^{-1}\right)$ and switching off the theoretical uncertainty on higher-order EW corrections.

require the resonance to be heavy enough so that Eq. (5) can be used to describe the Drell-Yan process in a particular invariant mass bin. In order to obtain bounds which are applicable for a variety of masses, we adopt the following procedure $[59,96]$ : We recalculate the projected $95 \%$ C.L. upper bound on $M / R$ by including only those invariant mass bins for which $m_{\ell \ell}<m_{\ell \ell \text { cut }}$. For a given $Z^{\prime}$ of mass $M$, a consistent bound on its coupling $R$ is obtained using $m_{\ell \ell \text { cut }}=\alpha M$, where $\alpha \lesssim 1 . \alpha$ can in principle depend on the width of the resonance, $\Gamma_{Z^{\prime}}$. The bounds on $M / R$ as a function of $m_{\ell \ell \text { cut }}$ are shown in Fig. 4. For small $m_{\ell \ell \text { cut }}$, the bound is weaker, while it saturates for $m_{\ell \ell \text { cut }} \gtrsim 3 \mathrm{TeV}$,

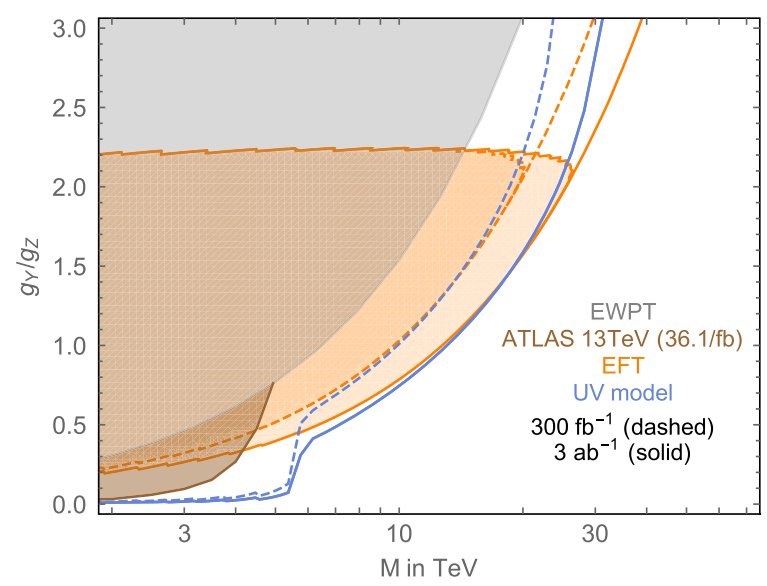

FIG. 5. Comparison between the $95 \%$ C.L. upper bound on $g_{Y}$ extracted using the EFT of Eq. (5) (orange lines), with $m_{\ell \ell \text { cut }}=$ $M-2.5 \times \Gamma_{Z^{\prime}}$ (orange shaded), and the full model of Eq. (5) (blue lines). The two bounds agree for masses $5.5 \mathrm{TeV} \lesssim M \lesssim$ $25 \mathrm{TeV}$. For smaller $M$, the EFT does not capture on-shell $Z^{\prime}$ production, and the bound extracted from the full model is much stronger. At larger masses and couplings, finite $Z^{\prime}$ width effects, which are not included in the EFT calculation, become important and lead to a weakening of the bound in the full model. The gray region shows the region which is excluded by low energy measurements. above which the energy growth of the partonic cross section is counterbalanced by the decrease of the parton luminosities. The right panel of Fig. 4 shows that an increase in the total integrated luminosity strengthens the bound only for $m_{\ell \ell \text { cut }} \gtrsim 1 \mathrm{TeV}$, since in this case the total uncertainty is dominated by the statistical uncertainty.

It is natural to ask how the bounds on a given $Z^{\prime}$ model, obtained from the full theory in Eq. (1), compare with those extracted from the EFT of Eq. (5). Using the hypercharge model as a benchmark, Fig. 5 shows the 95\% C.L. upper bound on the coupling $g_{Y}$, using the full model in Eq. (1). We compare this to the exclusion obtained from the EFT, where we choose either $m_{\ell \ell \text { cut }}=\infty$ or $m_{\ell \ell \text { cut }}=M-2.5 \times \Gamma_{Z^{\prime}}$.

Figure 5 shows that for small enough $M \lesssim 5.5 \mathrm{TeV}$, the EFT bound is much weaker than the one obtained from the full model. In this region, the cross section is dominated by on-shell $p p \rightarrow Z^{\prime}$ production, followed by $Z^{\prime} \rightarrow \ell^{+} \ell^{-}$ decay. The bound in this region approximates the reach of bump hunt searches, and we find a result consistent, within a factor of 2 in cross section, with prior bump hunt studies [35,38]. An order 1, difference is expected because our analysis uses a binned invariant mass spectrum that is designed to capture smooth interference effects, but is not optimized for narrow resonances. At larger masses, the bound on $g_{Y}$ agrees when using the full model versus the EFT. The agreement stops around $M \sim 25 \mathrm{TeV}$ and $g_{Y} / g_{Z} \gtrsim 2.5$. At large coupling, the $Z^{\prime}$ width is correspondingly larger, and $\Gamma_{Z^{\prime}} / M$ corrections become important. These lead to a cancellation in the size of the deviation from the SM prediction (see the red curve in Fig. 2).

Here we have focused on $2 \sigma$ exclusions. When $M \gtrsim 5.5 \mathrm{TeV}$, we find that a $5 \sigma$ discovery is not possible at the LHC, given LEP bounds. However, it is possible to have a signal with $3 \sigma$ significance. Additional 95\% C.L. projections for a $p p$ collider with a larger center-of-mass energy (27 and $100 \mathrm{TeV}$ ) are shown in the Supplemental Material. 
Conclusions. - In this Letter, we have shown that precision measurements of the shape of the dilepton invariant mass spectrum have broad reach to probe off-shell $Z^{\prime} \mathrm{s}$, extending the mass reach of direct searches. Unlike bump hunts, off-shell interference is insensitive to the presence of other decay modes. Our results only rely on the invariant mass distribution, but it would be interesting to explore how much sensitivity is gained by also using angular information. We have demonstrated significant reach for $Z$ 's, after a careful accounting of theoretical uncertainties. In order to fully realize this reach, our results motivate a concerted effort to control experimental uncertainties in energetic dilepton tails. The LHC may retain significant power, even if new physics is too heavy for direct production.

We thank Bogdan Dobrescu, Stefano Forte, Frank Petriello, Eram Rizvi, Tom Rizzo, and Hwidong Yoo for helpful discussions. We thank Christian Bauer for providing code that calculates the resummed EW corrections from Ref. [97]. S. A. acknowledges support by the COFUND Fellowship under Grant Agreement No. PCOFUND-GA2012-600377. M. F. is supported in part by DOE Grant No. DE-SC0010008. D. P. and J. T. R. are supported by NSF CAREER Grant No. PHY-1554858. This work was supported in part by the hospitality of the Aspen Center for Physics, which is supported by National Science Foundation Grant No. PHY-1066293.

*simone.alioli@unimib.it

†farina.phys@gmail.com

*duccio.pappadopulo@gmail.com

§ruderman@nyu.edu

[1] P. Langacker, R. W. Robinett, and J. L. Rosner, Phys. Rev. D 30, 1470 (1984).

[2] D. London and J. L. Rosner, Phys. Rev. D 34, 1530 (1986).

[3] G. Altarelli, B. Mele, and M. Ruiz-Altaba, Z. Phys. C 45, 109 (1989); 47, 676(E) (1990).

[4] A. Leike, Phys. Rep. 317, 143 (1999).

[5] J. Erler, P. Langacker, and T.-J. Li, Phys. Rev. D 66, 015002 (2002).

[6] M. Dittmar, A.-S. Nicollerat, and A. Djouadi, Phys. Lett. B 583, 111 (2004).

[7] M. Carena, A. Daleo, B. A. Dobrescu, and T. M. P. Tait, Phys. Rev. D 70, 093009 (2004).

[8] A. Freitas, Phys. Rev. D 70, 015008 (2004).

[9] T. G. Rizzo, arXiv:hep-ph/0610104.

[10] P. H. Chankowski, S. Pokorski, and J. Wagner, Eur. Phys. J. C 47, 187 (2006).

[11] J. D. Wells, arXiv:0803.1243.

[12] T. G. Rizzo, J. High Energy Phys. 08 (2009) 082.

[13] L. Ye, F. Petriello, and S. Quackenbush, Phys. Rev. D 80, 055018 (2009).

[14] J. Erler, P. Langacker, S. Munir, and E. Rojas, J. High Energy Phys. 08 (2009) 017.

[15] R. Diener, S. Godfrey, and T. A. W. Martin, arXiv:0910.1334.

[16] F. del Aguila, J. de Blas, and M. Perez-Victoria, J. High Energy Phys. 09 (2010) 033.
[17] J. Erler, P. Langacker, S. Munir, and E. Rojas, J. High Energy Phys. 11 (2011) 076.

[18] C.-W. Chiang, N. D. Christensen, G.-J. Ding, and T. Han, Phys. Rev. D 85, 015023 (2012).

[19] T. Han, P. Langacker, Z. Liu, and L.-T. Wang, arXiv: 1308.2738

[20] E. Accomando, A. Belyaev, J. Fiaschi, K. Mimasu, S. Moretti, and C. Shepherd-Themistocleous, J. High Energy Phys. 01 (2016) 127.

[21] B. Fuks and R. Ruiz, J. High Energy Phys. 05 (2017) 032.

[22] A. Greljo and D. Marzocca, Eur. Phys. J. C 77, 548 (2017).

[23] P. Langacker, Rev. Mod. Phys. 81, 1199 (2009).

[24] C. Patrignani et al. (Particle Data Group), Chin. Phys. C 40, 100001 (2016).

[25] G. Aad et al. (ATLAS Collaboration), J. High Energy Phys. 11 (2012) 138.

[26] S. Chatrchyan et al. (CMS Collaboration), Phys. Lett. B 720, 63 (2013).

[27] G. Aad et al. (ATLAS Collaboration), Phys. Rev. D 90, 052005 (2014).

[28] V. Khachatryan et al. (CMS Collaboration), J. High Energy Phys. 04 (2015) 025.

[29] V. Khachatryan et al. (CMS Collaboration), Phys. Lett. B 768, 57 (2017).

[30] M. Aaboud et al. (ATLAS Collaboration), Phys. Lett. B 761, 372 (2016).

[31] M. Aaboud et al. (ATLAS Collaboration), J. High Energy Phys. 10 (2017) 182.

[32] S. Chatrchyan et al. (CMS Collaboration), arXiv:1307 .7135 .

[33] G. Aad et al. (ATLAS Collaboration), arXiv:1307.7292.

[34] D. Hayden, R. Brock, and C. Willis, arXiv:1308.5874.

[35] S. Godfrey and T. Martin, arXiv:1309.1688.

[36] Y. Gershtein et al., arXiv:1311.0299.

[37] D. Pappadopulo, A. Thamm, R. Torre, and A. Wulzer, J. High Energy Phys. 09 (2014) 060.

[38] A. Thamm, R. Torre, and A. Wulzer, J. High Energy Phys. 07 (2015) 100.

[39] T. Golling et al., CERN Yellow Report No. 3, 2017.

[40] T. Appelquist, B. A. Dobrescu, and A. R. Hopper, Phys. Rev. D 68, 035012 (2003).

[41] A. Ferroglia, A. Lorca, and J. J. van der Bij, Ann. Phys. (Berlin) 16, 563 (2007).

[42] E. Salvioni, G. Villadoro, and F. Zwirner, J. High Energy Phys. 11 (2009) 068.

[43] E. Salvioni, A. Strumia, G. Villadoro, and F. Zwirner, J. High Energy Phys. 03 (2010) 010.

[44] L. Basso, S. Moretti, and G. M. Pruna, J. High Energy Phys. 08 (2011) 122.

[45] L. Basso, A. Belyaev, S. Moretti, and C. H. ShepherdThemistocleous, Phys. Rev. D 80, 055030 (2009).

[46] L. Basso, A. Belyaev, S. Moretti, and G. M. Pruna, Phys. Rev. D 81, 095018 (2010).

[47] L. Basso, A. Belyaev, S. Moretti, G. M. Pruna, and C. H. Shepherd-Themistocleous, Eur. Phys. J. C 71, 1613 (2011).

[48] L. Basso, S. Moretti, and G. M. Pruna, Phys. Rev. D 82, 055018 (2010).

[49] L. Basso, S. Moretti, and G. M. Pruna, Phys. Rev. D 83, 055014 (2011).

[50] B. Kors and P. Nath, Phys. Lett. B 586, 366 (2004). 
[51] B. Kors and P. Nath, J. High Energy Phys. 12 (2004) 005.

[52] B. Kors and P. Nath, J. High Energy Phys. 07 (2005) 069.

[53] M. Dittmar, Phys. Rev. D 55, 161 (1997).

[54] F. Petriello and S. Quackenbush, Phys. Rev. D 77, 115004 (2008).

[55] E. Accomando, A. Belyaev, L. Fedeli, S. F. King, and C. Shepherd-Themistocleous, Phys. Rev. D 83, 075012 (2011).

[56] E. Accomando, D. Becciolini, A. Belyaev, S. Moretti, and C. Shepherd-Themistocleous, J. High Energy Phys. 10 (2013) 153.

[57] V. Cirigliano, M. Gonzalez-Alonso, and M. L. Graesser, J. High Energy Phys. 02 (2013) 046.

[58] J. de Blas, M. Chala, and J. Santiago, Phys. Rev. D 88, 095011 (2013).

[59] M. Farina, G. Panico, D. Pappadopulo, J. T. Ruderman, R. Torre, and A. Wulzer, Phys. Lett. B 772, 210 (2017).

[60] A. Falkowski, M. Gonzlez-Alonso, and K. Mimouni, J. High Energy Phys. 08 (2017) 123.

[61] D. Rainwater and T. M. P. Tait, Phys. Rev. D 75, 115014 (2007).

[62] D. S. M. Alves, J. Galloway, J. T. Ruderman, and J. R. Walsh, J. High Energy Phys. 02 (2015) 007.

[63] W. Altmannshofer, P. J. Fox, R. Harnik, G. D. Kribs, and N. Raj, Phys. Rev. D 91, 115006 (2015).

[64] N. Raj, Phys. Rev. D 95, 015011 (2017).

[65] R. M. Capdevilla, A. Delgado, A. Martin, and N. Raj, arXiv: 1709.00439 [Phys. Rev. D (to be published)].

[66] S. Matsumoto, S. Shirai, and M. Takeuchi, arXiv:1711.05449.

[67] See the Supplemental Material at http://link.aps.org/ supplemental/10.1103/PhysRevLett.120.101801 for details on the calculations of the SM predictions, projections for future colliders, and comparisons with existing contact operator bounds, which also includes Refs. [68] and [69].

[68] J. Butterworth et al., J. Phys. G 43, 023001 (2016).

[69] A. Manohar, P. Nason, G. P. Salam, and G. Zanderighi, Phys. Rev. Lett. 117, 242002 (2016).

[70] R. Barbieri, A. Pomarol, R. Rattazzi, and A. Strumia, Nucl. Phys. B703, 127 (2004).

[71] G. Cacciapaglia, C. Csaki, G. Marandella, and A. Strumia, Phys. Rev. D 74, 033011 (2006).

[72] R. Hamberg, W. L. van Neerven, and T. Matsuura, Nucl. Phys. B359, 343 (1991); B644, 403(E) (2002).

[73] C. Anastasiou, L. J. Dixon, K. Melnikov, and F. Petriello, Phys. Rev. Lett. 91, 182002 (2003).

[74] C. Anastasiou, L. J. Dixon, K. Melnikov, and F. Petriello, Phys. Rev. D 69, 094008 (2004).
[75] K. Melnikov and F. Petriello, Phys. Rev. D 74, 114017 (2006).

[76] S. Catani, L. Cieri, G. Ferrera, D. de Florian, and M. Grazzini, Phys. Rev. Lett. 103, 082001 (2009).

[77] R. Gavin, Y. Li, F. Petriello, and S. Quackenbush, Comput. Phys. Commun. 182, 2388 (2011).

[78] F. A. Berends, R. Kleiss, J. P. Revol, and J. P. Vialle, Z. Phys. C 27, 155 (1985).

[79] U. Baur, S. Keller, and W. K. Sakumoto, Phys. Rev. D 57, 199 (1998).

[80] U. Baur, O. Brein, W. Hollik, C. Schappacher, and D. Wackeroth, Phys. Rev. D 65, 033007 (2002).

[81] C. M. Carloni Calame, G. Montagna, O. Nicrosini, and A. Vicini, J. High Energy Phys. 10 (2007) 109.

[82] A. Arbuzov, D. Bardin, S. Bondarenko, P. Christova, L. Kalinovskaya, G. Nanava, and R. Sadykov, Eur. Phys. J. C 54, 451 (2008).

[83] S. Dittmaier and M. Huber, J. High Energy Phys. 01 (2010) 060.

[84] Y. Li and F. Petriello, Phys. Rev. D 86, 094034 (2012).

[85] J. Alwall, R. Frederix, S. Frixione, V. Hirschi, F. Maltoni, O. Mattelaer, H. S. Shao, T. Stelzer, P. Torrielli, and M. Zaro, J. High Energy Phys. 07 (2014) 079.

[86] U. Baur, Phys. Rev. D 75, 013005 (2007).

[87] G. Bell, J. H. Kuhn, and J. Rittinger, Eur. Phys. J. C 70, 659 (2010).

[88] T. Becher and X. Garcia i Tormo, Phys. Rev. D 88, 013009 (2013).

[89] M. Ciafaloni, P. Ciafaloni, and D. Comelli, Phys. Rev. Lett. 84, 4810 (2000).

[90] M. Ciafaloni, P. Ciafaloni, and D. Comelli, Nucl. Phys. B589, 359 (2000).

[91] M. Ciafaloni, P. Ciafaloni, and D. Comelli, Phys. Lett. B 501, 216 (2001).

[92] M. Ciafaloni, P. Ciafaloni, and D. Comelli, Phys. Rev. Lett. 87, 211802 (2001).

[93] A. Manohar, B. Shotwell, C. Bauer, and S. Turczyk, Phys. Lett. B 740, 179 (2015).

[94] V. Khachatryan et al. (CMS Collaboration), Eur. Phys. J. C 75, 147 (2015).

[95] G. Aad et al. (ATLAS Collaboration), J. High Energy Phys. 08 (2016) 009.

[96] S. Alioli, M. Farina, D. Pappadopulo, and J. T. Ruderman, J. High Energy Phys. 07 (2017) 097.

[97] C. W. Bauer and N. Ferland, J. High Energy Phys. 09 (2016) 025 . 